\section{Negociando el derecho a la ciudad: grafiti en Bogotá}

\section{Negotiating the right to the city: graffiti in Bogota}

\section{Alba Griffin*}

\section{Resumen}

El concepto lefebvriano del derecho a la ciudad es una denuncia de las realidades de exclusión y alienación en la sociedad urbana, y una exigencia para la justicia social a través de la participación en el espacio público urbano y su apropiación. Al aplicar este concepto al contexto contemporáneo del grafiti en Bogotá, Colombia, la intención del artículo es demostrar como un análisis alineado con la idea de una geopolítica de lo cotidiano expone las prácticas políticas y cotidianas de los habitantes urbanos. El derecho a la ciudad no es algo que simplemente exigen

Departamento de Lenguas Modernas, Facultad de Humanidades y Ciencias Sociales, Newcastle University, UK alba.griffin@newcastle. ac.uk los grafiteros, es un derecho negociado con el gobierno local, contribuyendo a la legitimación de la práctica creativa. Sin embargo, los grafiteros también enfrentan la reproducción de exclusión en la vida cotidiana y los límites del derecho a la ciudad se revelan en una jerarquía estética, que a su vez reproduce una jerarquía social.

Palabras claves: grafiti, Bogotá, espacio urbano, derecho a la ciudad

\begin{abstract}
The Lefebvrean concept of the right to the city denouncestherealities of exclusionandalienation in urban society and demands social justice through the participation in, and appropriation of, urban space. Applying this concept to the contemporary context of graffiti in Bogota, this article aims to show how an analysis in line with an everyday geopolitics offers an insight into the everyday political practices performed by urban inhabitants. The right to do graffiti in the city is not simply demanded by graffiti artists but negotiated with the local government in Bogota, and this negotiation has both stemmed from, and contributed to, a legitimisation of this creative practice. However, graffiti artists also face the reproduction of processes of exclusion in everyday life and the limits of the right to the city are revealed through an aesthetic hierarchy that reproduces a social hierarchy.
\end{abstract}

Key words: graffiti, Bogota, urban space, right to the city 


\section{Introducción}

Bogotá es una ciudad pintada, pero no por el color de sus fachadas sino por las inscripciones, los dibujos y los mensajes que aparecen en sus paredes. Cuando se habla sobre el grafiti de Bogotá, hay que tener en cuenta cuatro tipos de grafiti que aparecen mayormente en la ciudad de acuerdo con la elaboración de Castro Pulido. ${ }^{1}$ El grafiti writing es un estilo asociado con el movimiento global de hip-hop y las expresiones de jóvenes que tradicionalmente han sido marginalizados en las grandes urbes (imagen 1). Buscan reconocimiento y visibilidad a través de nombres o tags elaborados en estilos particulares de su subcultura, cubriendo la ciudad con estos a través de una actividad denominada bombing. ${ }^{2}$ El grafiti barrista tiene en común la elaboración de nombres en estilos difíciles de leer, pero se enfoca más en difundir los nombres de agrupaciones de aficionados al fútbol para marcar territorios (imagen 2). Por su estilo hay una influencia de la pixação brasileña y la tipografía utilizada por los grupos de música metal. Ambos, el grafiti writing y el grafiti barrista, son visibles por toda la ciudad, predominando la presencia de estos en los barrios que se encuentran fuera de la ciudad. En cambio, en el centro de la ciudad, además de estos tipos de grafiti hay una diversidad de consigna y de arte urbano (imagen 3 y 4). El grafiti de consigna tiene una larga historia en la región y representa la comunicación contestataria de movimientos universitarios, sindicalistas, juveniles y de oposición. Utilizan las paredes para difundir

Castro Pulido, S. Diagnóstico Graffiti Bogotá 2012. Informe Final para el Instituto Distrital de las Artes en Bogotá, Colombia.

El grafiti writing y el movimiento hip-hop tienen sus orígenes en Filadelfia y Nueva York de los Estados Unidos. De ahí vienen la terminología que se usan en Bogotá, incluidos el writing, tags y bombing. mensajes críticos o propaganda, mostrando las fallas en el sistema y el descontento de los ciudadanos. El centro de la ciudad es ideal para el grafiti de consigna porque en este se concentran las instituciones del poder y los representantes del Estado a quienes están dirigidos los mensajes. El arte urbano también busca visibilidad, pero no necesariamente con mensajes críticos, es una manera de participar en la cultura visual de la ciudad, jugando con la materialidad del espacio a través de murales, esténciles, stickers, posters, entre otros, democratizando de esta forma las esferas del arte y la esfera del espacio público (Wacławek 2011). Mientras que estas definiciones subrayan las diferencias entre distintos tipos de grafiti, en este ensayo el término "grafiti" se utiliza para referirse a todos los tipos, para discutir lo que tienen en común: que todos los que los hacen están reclamando el derecho a la ciudad.

El derecho a la ciudad se refiere al concepto y proyecto elaborado principalmente por Henri Lefebvre en la Europa de los años 60. Sin embargo, tiene mucha resonancia por todo el mundo gracias a su intención de revelar y combatir la inequidad social concomitante con los espacios urbanos. Una de las ideas fundamentales es que los que habitan la ciudad los subalternos, las clases trabajadoras - deben tomar las decisiones para transformar la ciudad y deben participar en la producción de esta. Según Lefebvre, la apropiación del espacio urbano por parte de estos grupos sociales indicaría una ciudad más democrática. Hasta cierto punto, por lo menos en el nivel simbólico, los grafiteros exigen su derecho a la ciudad porque reclaman visiblemente lo que ven como su derecho a pintar la ciudad. Además, en Bogotá esta exigencia ha ganado cierta formalidad a través de la ley y las mesas de grafiti, donde la administración 
local dialoga con la comunidad de grafiteros. Como demuestro en el análisis siguiente, el contexto bogotano sugiere una apertura política hacia estas expresiones culturales y el Estado pretenda negociar con los grafiteros y no solo sancionar la práctica. Sin embargo, el tema de apropiación, fundamental para el concepto del derecho a la ciudad, da mayor complejidad a las dinámicas de grafiti en la ciudad. Los artistas no solo participan en negociaciones con el Estado, sino reclaman su derecho de construir la ciudad a su manera. Aquí se encuentra una tensión más conflictiva, porque el Estado, y los otros habitantes de la ciudad, parecen distinguir entre los grupos sociales que tengan un derecho legítimo a la ciudad, y los otros que no, reforzando los límites de la participación y la apropiación.

Por lo tanto, la relación entre el grafiti y el derecho a la ciudad merece un análisis más a fondo en cuanto demuestra las complejas interacciones y negociaciones entre el Estado y los ciudadanos, la complejidad del significado de la participación y la apropiación de la ciudad. Tal análisis requiere una posición conceptual más enfocada en la cotidianeidad y en los puntos de vista de los que habitan la ciudad.

Siguiendo la dirección del dossier para identificar una "geopolítica de lo cotidiano", el artículo prioriza las perspectivas de los que hacen grafiti para entender sus propias visiones del espacio urbano, el significado simbólico de sus prácticas estéticas y sus formas de participar políticamente en la escala cotidiana. En consideración de los puntos de vista de los grafiteros bogotanos, entrevistados por la autora en el 2015 y 2016, la mayor parte del presente ensayo tiene como objetivo explorar estos temas, así como las complejidades y contradicciones que surgen, mediante sus experiencias de pintar la ciudad. Para iniciar se presenta un resumen que contextualiza el derecho a la ciudad y la geopolítica de la cotidianeidad en el sentido utilizado para esteartículo, con atención particular a los paralelos entre los dos campos en cuanto al enfoque en los sujetos, espacios y escalas tradicionalmente excluidos de consideraciones políticas y la explícita motivación de ver el análisis académico como parte de un proyecto político. Después, considero las dinámicas de grafiti en Bogotá concomitante con el derecho a la ciudad, enfocando el análisis en las experiencias individuales de los artistas para exponer lo político de esta práctica cultural.

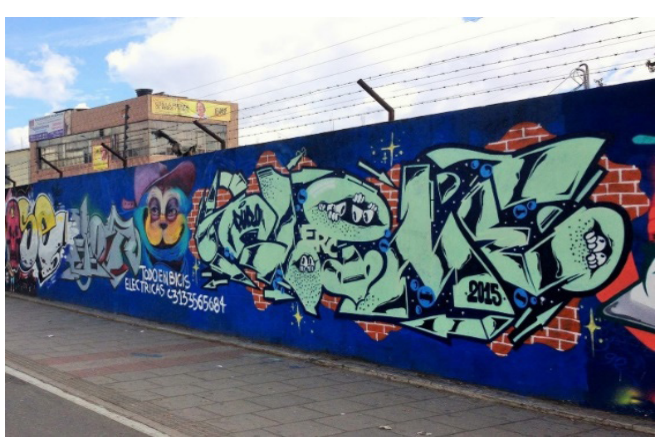

Imagen 1. Grafiti writing (fuente: autora)

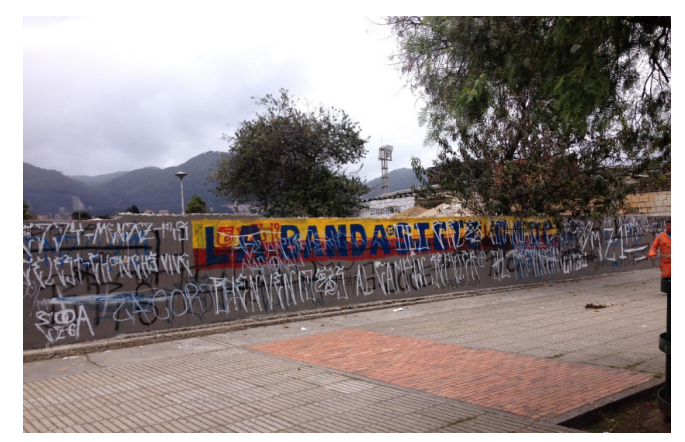

Imagen 2. Grafiti de barrista (fuente: autora) 


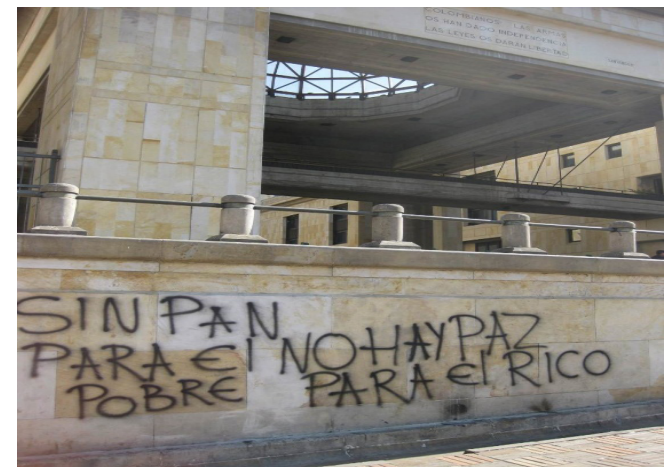

Imagen 3. Grafiti de consigna (fuente: autora)

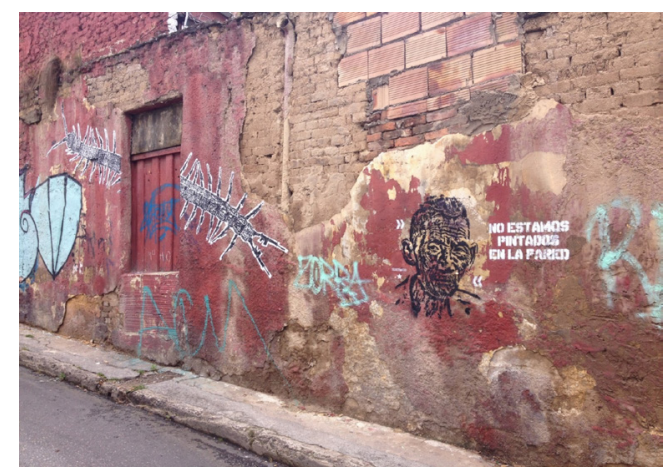

Imagen 4. Arte urbano (fuente: autora)

\section{El derecho a la ciudad y la geopolítica de lo cotidiano}

El concepto del derecho a la ciudad es notorio por ser algo confuso, impreciso y ambiguo. Dice Peter Marcuse que es un slogan "inmediatamente comprensible e instintivamente atractivo" pero al mismo tiempo una formulación "provocativa y teóricamente compleja" (Marcuse 2012b: 29). ${ }^{3}$ Para Mark Purcell, el derecho a la ciudad

"both an immediately understandable and intuitively compelling slogan, and a theoretically complex and provocative formulation" (todas las traducciones por la autora) es subdesarrollado en cuanto a su aspecto teórico y político, pero sigue siendo "fascinante y desconcertante" (Purcell 2002: 100). ${ }^{4}$ Esta imprecisión subraya la dificultad de implementar esta idea, dado que depende de cómo se define el derecho, pero al mismo tiempo su fuerza como concepto radical y amplio es evidente en la posibilidad de utilizarlo para "iluminar las conexiones entre varias luchas por la justicia social" (Attoh 2011: 670). ${ }^{5}$ Indudablemente, es un concepto productivo: como dice Marcuse, "en manos de Henri Lefebvre [...] se dota de una intención revolucionaria tanto en la teoría social como en la práctica política" (Marcuse 2012a: 17-18). Sería provechoso, entonces, conceptualizar el derecho a la ciudad como un proyecto político en curso en vez de un derecho fijo, y que está basado en las prácticas existentes y en las futuras, al igual que en las luchas por la justicia social.

En la obra de Lefebvre y en el campo de estudios urbanos críticos (CriticalUrbanStudies), elavance hacia el derecho a la ciudad empieza con el propósito de indagar en los procesos espaciales para entender las dinámicas sociopolíticas y las relaciones de poder (Brenner et al. 2012). Para Lefebvre, el problema con muchas ciudades contemporáneas es la violencia de la desigualdad social como consecuencia de la hegemonía del capitalismo; la ciudad se ha convertido en sitio de empobrecimiento de forma material, creativa y emocional (Lefebvre 2014; Lefebvre et al. 1996). La vida cotidiana para los subalternos dentro de

"theoretically and politically underdeveloped" but is "at once exciting and disconcerting"

5 "On the one hand, the radical openness of the right to the city concept can serve to illuminate connections between varying struggles for social justice. On the other hand, it is hard to square this radical openness with the notion that the practical significance of rights depends both on how we define a right and on the specific form a given right takes" 
sistemas capitalistas se encuentra enmarcada por la exclusión material y alienación espiritual. Esta situación se ve reflejada en diferentes problemáticas como, por ejemplo, la falta de acceso a la vivienda, el empleo y la educación, así como a actividades creativas, a la expresión libre de género, de religión o de la sexualidad. El reconocimiento de la exclusión y alienación como formas de opresión es significativo porque reclama la atención a la pregunta de quiénes tienen el derecho a la ciudad, entendido como los beneficios de los espacios urbanos. La perspectiva de Lefebvre estaba muy ligada a las clases sociales, así que para él los más excluidos venían de la clase obrera (Busquet 2013). Sin embargo, lo subalterno no describe un grupo social fijo, es una posición que describe a los que no tienen poder en contextos históricos específicos (Crehan 2016: 16-17). Los límites actuales al derecho a la ciudad no solo tienen que ver con una conceptualización de subalternos en cuanto a la exclusión y desigualdad material, sino también otras formas culturales de subalternidad. Esto es revelador porque investigar las relaciones de poder y cómo se manifiestan en la vida urbana requiere una mayor atención hacia las maneras en la que la gente se encuentra excluida y alienada. El punto clave, el problema que el derecho a la ciudad quiere enfrentar, es que los ciudadanos son los que habitan la ciudad, pero no tienen control sobre este espacio.

\section{Puesto que reconoce las necesidades} económicas y materiales, pero también las necesidades culturales, el enfoque del derecho a la ciudad es total, no parcial, y requiere una revolución urbana en cuanto a la producción del espacio. Aunque larga, la declaración de David Harvey es perspicaz porque refleja la amplitud del derecho a la ciudad:

\begin{abstract}
La cuestión de qué tipo de ciudad queremos no puede separarse del tipo de personas que queremos ser, el tipo de relaciones sociales que pretendemos, las relaciones con la naturaleza que apreciamos, el estilo de vida que deseamos y los valores estéticos que respetamos. El derecho a la ciudad es por tanto mucho más que un derecho de acceso individual o colectivo a los recursos que esta almacena o protege; es un derecho a cambiar y reinventar la ciudad de acuerdo con nuestros deseos (Harvey 2013:20).
\end{abstract}

Esta revolución tiene que beneficiar, y venir de, los que habitan la ciudad en vez de los que la planean y, por consiguiente, dos nociones esenciales vinculadas al proyecto son la participación y la apropiación. Harvey señala la importancia del "ejercicio de un poder colectivo", de la participación de los ciudadanos en las decisiones que les afecten, en las decisiones sobre el espacio urbano y la vida urbana. En teoría, la posibilidad de participación viene con el modelo democrático y los derechos asociados con la ciudadanía, pero como dice Harvey, es una libertad "descuidada". En sociedades dominadas por el neoliberalismo y el capitalismo, la priorización del valor de cambio toma precedencia sobre el valor de uso. Esto quiere decir que los que ahora tienen el derecho a la ciudad son las elites políticas y económicas, quienes toman decisiones sobre el espacio urbano basadas en la acumulación capitalista y la mercantilización. La participación política de los habitantes del espacio urbano es mediada por estas elites, no es directa, como la conceptualiza Lefebvre, aunque como veremos, las maneras en que la gente reclama su derecho a la ciudad demuestran la negociación de esta situación a través de "otras" acciones políticas. Vinculado a la acción política no tradicional está el concepto de apropiación, que va más allá de la participación en los procesos de urbanización, porque refleja los cambios más amplios y estructurales propuestos por Lefebvre (Purcell 
2002). La apropiación del derecho a la ciudad por parte de los habitantes sería el revés de la primacía del valor de cambio, ya que prioriza el valor de uso - es el reconocimiento de "nuestros deseos" y la ciudad que "queremos" como colectivo urbano. Esta propuesta significa no solo que los que utilizan el espacio deben tener el derecho de utilizarlo, sino que también toman control sobre la producción del espacio urbano en cuanto a las decisiones políticas tanto como las decisiones estéticas, en el presente y en el futuro. Ejemplos de la apropiación del espacio incluyen acciones y/o formas de protesta tan variadas como el movimiento "Occupy", ${ }^{6}$ vendedores ambulantes, la parcial $\mathrm{o}$ no legalidad de barrios autoconstruidos y, como argumentaré, el grafiti.

Aunque el derecho a la ciudad sea un proyecto político utópico, dada la impresionante escala de cambios estructurales necesarios, a Lefebvre le interesa la dimensión de lo cotidiano. Su conceptualización de la cotidianeidad revela tanto la reproducción de relaciones sociales desiguales como la producción de resistencia en lo que llama "lived space" (Lefebvre 2008). En este espacio vivido hay una confluencia de los procesos a gran escala que organizan y construyen espacio; la ideología que guía la representación y construcción de espacios y las prácticas en la vida cotidiana de los habitantes del espacio. Para entender las dinámicas urbanas hay que entender cómo viven los habitantes de la ciudad y cómo responden al poder, una perspectiva que refleja la geopolítica de lo cotidiano. Seguidamente, hay una elaboración de lo que una perspectiva basada

El movimiento Occupy se refiere a las protestas organizadas en ciudades por todo el mundo para criticar la desigualdad social y política, pero destacan las apropiaciones de lugares simbólicos de la ciudad capitalista, por ejemplo, Wall Street en Nueva York. en la geopolítica de lo cotidiano ofrece a la forma en que podemos investigar el derecho a la ciudad, sobre todo en cuanto a la perspectiva y agencia de los "subalternos"; al espacio de lo cotidiano; y a la motivación política detrás de ver la geopolítica de otra manera.

Lefebvre dice que cualquier proyecto para reclamar el derecho a la ciudad y que apunte a mejorar la producción del espacio hacia una producción más igualitaria, tiene que venir desde los que habitan la ciudad y no los que la planean (Lefebvre et al. 1996: 158). Es por esto que resulta necesario identificar los problemas y los procesos de resistencia a través de las perspectivas de los ciudadanos, de los subalternos que habitan y utilizan el espacio. La imbricación de la política del Estado con la vida cotidiana, además de las relaciones de poder y su presencia en diferentes espacios urbanos, resaltan la necesidad de reconocer la variedad de sujetos afectados por, y participantes en, la geopolítica. Para conceptualizar la relación entre la geopolítica y lo cotidiano, Rachel Pain y Susan Smith subrayan la contribución de la crítica feminista y, en particular, su propósito de reconsiderar y revaluar los sujetos, espacios y escalas incluidos en el estudio geopolítico (Pain y Smith 2008: 6). Una geopolítica feminista no representa una sola perspectiva ni un interés exclusivo en las cuestiones de género, sino que intenta animar un análisis más complejo en cuanto a la crítica teórica de la geopolítica - preguntando quienes producen la geopolítica (Koopman 2011; Hyndman 2004). Tradicionalmente, la investigación geopolítica no ha prestado atención a las perspectivas de grupos sociales particulares, por ejemplo mujeres y jóvenes (Dowler y Sharp 2001). Una investigación de las perspectivas y experiencias de esta variedad de sujetos ofrece diferentes 
maneras de ver las realidades geopolíticas en una ciudad como Bogotá. Pero en vez de solo verlos como objetos de investigación, hay que verlos como sujetos que producen conocimiento. Esa es la crítica de Skelton, que ofrece un análisis sobre las perspectivas y las prácticas políticamente significativas de las y los jóvenes. Argumenta que el poder y los procesos políticos (desde la gran escala hasta lo más personal/cotidiano) no solo afectan a diferentes grupos sociales sino que estos grupos pueden afectar la conceptualización del poder y de la política (Skelton 2010).

Poner atención a la variedad de sujetos que son políticamente activos también requiere una reevaluación de los espacios y las escalas de la esfera geopolítica. El derecho a la ciudad, y sus límites, se visibilizan a través de las prácticas y posibilidades de habitar la ciudad en la vida cotidiana. Las prácticas políticas de los habitantes no necesariamente se alinean con una visión normativa de lo que es la participación política. Para Sara Koopman, el concepto de alter-geopolitics va más allá de la geopolítica crítica, anti y feminista, porque reconoce las actividades cotidianas y de escala local no solo como sitios de investigación académica, sino como la geopolítica en práctica (Koopman 2011). Su enfoque está en las actividades de movimientos sociales, pero se aplica a los jóvenes descritos por Skelton y otros, quienes forman percepciones de política y ciudadanía a través de sus relaciones con el espacio y la gente en su entorno, y actúan en estos contextos (Skelton 2010; Hörschelmann y El Rafaie 2014; Kallio et al. 2016). Patiño, Alvarado y Ospina-Alvarado ofrecen una investigación sobre los jóvenes de Colombia y sus prácticas políticas, destacando que son conceptualizados como objetos pasivos. Sin embargo, los jóvenes tienen sus propias formas de hacer política, muchas veces separándose de las elites e instituciones hegemónicas por su desencanto y falta de confianza en ellas. Más bien, las prácticas cotidianas de los jóvenes, su participación en comunidades, en movimientos sociales y actividades culturales reflejaban sus perspectivas y acciones geopolíticas (Patiño et al. 2014). Cuando se reconoce que los jóvenes hacen política, pero "a su manera" y en la vida cotidiana, se puede ver lo político de hacer grafiti, manifestado principalmente a través de su participación en, y apropiación de, los espacios públicos de la ciudad.

Antes de considerar los rasgos particulares del grafiti, hay un punto final en cuanto a la relación entre el derecho a la ciudad y la geopolítica de lo cotidiano que tiene que ver con una motivación explícitamente política. De acuerdo con Lefebvre, los ciudadanos (sobre todo los más marginalizados por clase, género, orientación sexual o etnia) están, en muchos casos, excluidos de los beneficios materiales, creativos y espirituales del espacio urbano. La ciudad es un nido de la heterogeneidad social, económica y política, y esta heterogeneidad (y sus desigualdades) se visibilizan forzosamente a través del espacio y los usos de este por sus habitantes (Sassen 2013). En la vida cotidiana, las elites financieras, políticas y sociales controlan las condiciones de uso del espacio urbano y las expectaciones de vida de la sociedad urbana, pero los ciudadanos o habitantes de la ciudad tienen sus propias tácticas para desafiar estas relaciones de poder (De Certeau 1980). La geopolítica de lo cotidiano plantea la obligación de entender las tácticas de los subalternos y las estrategias de las elites en contextos de poder, para evitar la suposición de que los sujetos sociales más subalternos son 
meramente pasivos o cómplices. Algo que Pain y Smith enfatizan es lo que describen como una prerrogativa moral para escuchar a las narrativas e interpretaciones personales de los sujetos en su vida diaria para descubrir soluciones a problemas políticos (Pain y Smith 2008: 10). ${ }^{7}$ Claro que hay procesos que impiden la acción o la resistencia, pero no quiere decir que no existan opciones alternativas. Las prácticas de resistencia en la vida cotidiana, las perspectivas alternativas y la agencia de los individuos, tienen relevancia no solo por sus mensajes específicos, sino porque son prácticas que proyectan una agenda política. Mientras que la geopolítica crítica no necesariamente se enfoca en las prácticas de resistencia, sino también en la reproducción de poder, Hyndman dice que la geopolítica feminista se distingue porque en vez de meramente criticar las relaciones de poder, enuncia su motivación política a través de un enfoque en otras maneras de ver y otras formas de estar y participar en el mundo (Hyndman 2004: 312). Una geopolítica de lo cotidiano, como se concibe aquí, necesita identificar el enlazamiento de poder en las prácticas espaciales de la sociedad urbana con el propósito político de valorar la resistencia. Al mismo tiempo, es necesario prestar atención a las complejas interacciones o grupos de poder que pueden detener la potencia de la sociedad urbana.

Esta perspectiva nos da algunas pistas para entender el grafiti como una articulación simbólica y política del derecho a la ciudad. Vale la pena resaltar la idea de que el derecho a la ciudad se reclama, no se ofrece, y que hay

"there is a moral prerogative to emphasise people's own accounts of the pattern of their emotional landscapes. Ordinary lives often hold the solution to some of the more intractable political problems." espacios de resistencia a través de las prácticas geopolíticas de lo cotidiano. En su discusión sobre las prácticas estéticas, incluyendo el grafiti, de la sociedad urbana, Armando Silva dice que tienen una cualidad proyectiva:

[1]levan implícito un deseo de cambio, y entonces su afirmación de clase o de ghetto hace manifiesto que se contraponen a otro orden, el que rechazan; de este modo sus recreaciones se 'ordenan' hacia una fantasía condenatoria (Silva 1987: 131).

De esto modo, el grafiti participa en el imaginario urbano de manera activa, reflejando una perspectiva y punto de vista crítico y político que promueve un cambio. El imaginario social se refiere a las prácticas, normas, imágenes, interacciones y verdades sociales que contribuyen a formar la idea y experiencia de hacer parte de una sociedad (Silva 2006). Los imaginarios sirven para reproducir el orden social, pero también revelan otras maneras de ver. Al reclamar su derecho a la ciudad los grafiteros representan un desafío a las múltiples exclusiones y alienaciones de la vida cotidiana al nivel del imaginario social. Sobre todo, insisten en su derecho de transformar la ciudad a través de sus propias formas de participación política y de su apropiación creativa del espacio público. Al seguir la línea de investigación de la geopolítica de lo cotidiano, estas formas de agencia y autonomía en y por el espacio urbano sobresalen como prácticas políticas cotidianas. Al mismo tiempo, un enfoque en las experiencias y las perspectivas de los que hacen grafiti resalta también la complejidad de las negociaciones necesarias entre la ciudadanía y el Estado, y los límites del derecho a la ciudad. 


\section{Negociando la participación en la ciudad}

Si el derecho a la ciudad significa el derecho de participar en las decisiones que transforman el espacio, ¿los grafiteros tienen el derecho a la ciudad en Bogotá? Con lo que concierne a la estética urbana, puede ser. El apoyo al grafiti por parte de la alcaldía está establecido en la Ley de Grafiti - Decreto N075 de 2013 y Decreto N529 de 2015. Estos decretos oficializan las reglas para controlar el grafiti y precisan en qué condiciones se puede practicar el grafiti conforme a la ley. A la vez, es una ley que refleja cómo el estatus de grafiti (sobre todo el writing $y$ el arte urbano) ha cambiado en el mundo contemporáneo hasta llegar a ser valorado, y aun vendido, como capital cultural que da valor al espacio (Schacter 2014). En particular, la ley en Bogotá reconoce todas las formas de grafiti como expresión cultural y promete que el gobierno "establecerá estímulos especiales y promocionará la creación, la actividad artística y cultural, la investigación y el fortalecimiento de las expresiones culturales". La promesa incluye el uso de espacios libres para pintar, fondos y convocatorias para proyectos que incentivan las habilidades y el desarrollo cultural de grafiti, además de la financiación de grandes obras de arte urbano para las cuales invita a participar a los grafiteros. La ley autoriza a pintar en las fachadas de la propiedad privada, lo que implica muchas veces una negociación entre el artista y el propietario sin que el gobierno intervenga. Otra característica notable es que la ley subraya las posibles sanciones contra los que hacen grafiti de manera ilegal - algo que no fue oficializado antes y que permitió mucha corrupción y violencia por parte de la policía. Hoy en día, el apoyo al grafiti se ve reflejado en los grandes medios de comunicación, que celebran la ciudad pintada, entrevistan a los artistas e incluso crean álbumes de fotos aplaudiendo la expresión cultural de los jóvenes bogotanos. El grafiti es un componente de la industria cultural, con galerías y tiendas independientes exhibiendo y vendiendo obras de grafiteros locales, incluso hay un tour de grafiti, el cual recibió la bendición explícita del anterior alcalde, Gustavo Petro, en Twitter.

El reconocimiento del grafiti, por lo menos como una práctica cultural y creativa, es valioso porque indica una posición política frente a la ciudad que reconoce los grupos sociales muchas veces marginalizados de forma material, y promueve su creatividad a través de la posibilidad de utilizar el espacio público para esta expresión cultural. Bogotá es una de las ciudades más grandes y desiguales en América Latina, con una población de 8 millones de habitantes y se prevé que llegará al estatus de mega-ciudad en $2030 .{ }^{8}$ La marginalización y la segregación social están trazadas sobre la organización espacial de la ciudad de forma material, pero también de forma simbólica. Una estratificación oficial de los distintos sectores de la ciudad indica los barrios pobres, de clase media y de lujo para señalar quienes necesitan subsidios, pero el efecto es un fortalecimiento de la segregación social, la exclusión y la alienación. El acceso al centro de la ciudad puede ser limitado para los que vienen de barrios marginalizados porque no "pertenecen" en el centro simbólico del poder económico, político y cultural. Igualmente, muchas personas de la clase más alta descubren el centro de la ciudad solo a través del empleo o la educación universitaria porque en sus barrios de lujo, con seguridad privada, resuelven todas sus necesidades (Thibert y Osorio 2014).

Según el informe "World Urbanization Prospects: The 2014 Revision" publicado por las Naciones Unidas. 
La valoración del uso cultural del espacio público, sobre todo por los que vienen de posiciones tradicionalmente contra-culturales y marginalizadas, corresponde con una tendencia política de propulsar un cambio más grande en cuanto al valor social del espacio urbano. Vale la pena recordar la declaración de Harvey en cuanto al derecho a la ciudad, que "la ciudad que queremos" se entrecruza con la sociedad a la que aspiramos o la sociedad que deseamos. Aquí el concepto del imaginario toma relevancia. Omar Rincón y María Paula Hoyos describen las políticas y acciones opuestas de los alcaldes en Bogotá en la historia reciente, señalando que reflejaban distintas posiciones frente a cómo mejorar la ciudad. Algunos de los eslóganes apuntan a sus discursos, todos aludiendo a una ciudad utópica: con Antanas Mockus fue "Bogotá: Para vivir todos del mismo lado", con Enrique Peñalosa era "Por la Bogotá que queremos" y después "Bogotá: Mejor para todos", Luis Eduardo Garzón proponía "Bogotá sin indiferencia" y Gustavo Petro prometió una "Bogotá Humana". Sin embargo, la pregunta relevante es ¿cuál es nuestra idea de la ciudad?, ¿qué es la sociedad deseada? y, sobre todo, ¿para quién y para qué hacer en la ciudad? En Bogotá, la segregación y la desigualdad se visibilizan en las actitudes e imaginarios políticos, los cuales están polarizados por clase social. En breve, el programa de Mockus lograba combatir la alienación a través de la cultura ciudadana que fomentaba un compromiso entre los ciudadanos y el espacio urbano. Peñalosa propulsó una transformación material de la ciudad con el desarrollo de su infraestructura, pero de manera tal que priorizó la privatización. En cambio, Garzón implementaba un programa para combatir las desigualdades y exclusiones materiales y simbólicas, una tendencia enfocada en lo social que fue continuada por Petro (Rincón y Hoyos 2013). Dado estas prioridades distintas, lo que predomina en cuanto al derecho a la ciudad es que en los imaginarios urbanos bogotanos hay una clara percepción del partidismo de los políticos: unos ignoran a ciertas clases o grupos sociales a favor de otros. Del mismo modo, el reconocimiento de la juventud y su desarrollo cultural han sido temas preponderantes para los que tienen programas sociales, aunque otros autores ven con cinismo los discursos y las acciones hacia los jóvenes porque están más enfocadas en el buen comportamiento de ellos y no su autonomía política y cultural (Sarmiento 2006; Muñoz 2000).

Además de reconocer las prioridades sociales en las distintas políticas de los alcaldes y partidos políticos en Bogotá, también es necesario resaltar que la inclusión en el espacio público de la ciudad no fue algo simplemente ofrecido a los que hacen grafiti. Como dice Lefebvre, el derecho a la ciudad es un grito de socorro y una exigencia (Harvey 2013: 10). Hay que reconocer la larga historia de grafiti en Bogotá y las acciones de sus creadores. Como demuestra Armando Silva en sus trabajos académicos investigando el grafiti en Colombia, su evolución está ligada a los movimientos sociales y estudiantiles que han utilizado el medio desde los años 60's (Silva 1989). El grafiti writing también tiene una larga historia en la ciudad, con la llegada en los años 80's y 90's de información sobre la subcultura y productos como videos, casetes y revistas, que fueron compartidos por grupos de jóvenes que acomodaron el significado del hip-hop estadounidense a su contexto local (Tickner 2008). Como dice el artista Skore, su iniciación en el mundo del grafiti se debió a su hermano mayor, quien le mostraba videos de rap. Dado que no entendía las canciones en inglés, se interesaba más por lo visual - el grafiti escrito en el fondo: 
[P]ausaba los videos en las partes donde veía grafiti, murales, y $[\ldots]$ empecé a copiarlos $[\ldots]$ intentar verlos $[\ldots]$ descifrarles o algo así [...] Esto me despertó el interés al grafiti. $^{9}$

También aparecían escuelas de grafiti como la Familia Ayara, fundada en 1996, donde artistas aprendieron de primera mano de las generaciones anteriores adentro de la subcultura. Cest es uno de estos artistas de grafiti y describe su experiencia:

[E]n Ayara fue que encontré la gente que le gustaba hacer lo mismo que yo. Entonces investigábamos, buscábamos videos, empecé a descubrir también mucho más la ciudad gracias a eso. ${ }^{10}$

Desde los años 2000, la ciudad ha visto un incremento no solo en el grafiti writing, sino también en el arte urbano, los esténciles y los artistas que intentan elaborar algo más pictórico en las paredes.

Por consiguiente, el desarrollo del grafiti y de la ciudad pintada vino desde las subculturas desde las prácticas cotidianas y creativas de los jóvenes urbanos. La llegada hasta el punto contemporáneo es, para Lili Cuca, un recorrido que demuestra la participación de los grafiteros en el desarrollo de Bogotá:

\begin{abstract}
Mañana el alcalde que siga puede llegar a pintar todos los muros de gris y se va a acabar, y punto final. Vamos a empezar a luchar otra vez contra eso. Pero no es ese el asunto. El asunto es todo el camino que ha sido recorrido, los números de personas que pintaron, los materiales que llegaron [...] los que se pintaban antes, las dificultades que se tuvieron para pintar antes, y la manera como se genera ese tipo de cosas. ${ }^{11}$
\end{abstract}

\footnotetext{
Entrevista con la autora

10 Entrevista con la autora

11 Entrevista con la autora
}

Aún si hay apoyo político ahora, hay que reconocer que fueron los grafiteros quienes impulsaron la transformación de la imagen del grafiti y de las relaciones entre ellos y el Estado. Lili Cuca continúa:

Me resulta a mí, altamente importante reconocer que
tenemos ya una historia en esto y que las personas que
están realizando las nuevas prácticas deben tener ese
conocimiento previo [...] para que sepan qué ha pasado,
que hay mucha gente que se quemó el cuello antes [...]
se sentó muchas veces a hablar luego, de cómo generar
el cambio adentro de la ciudad para que el grafiti dejara
de ser un problema, que se había convertido en que se
viera como un aporte a la cultura y a la identidad nacional.

Reconocer la participación activa de los grafiteros es primordial porque la creación de la ley de grafiti inicialmente siguió la línea de ver el grafiti o como algo vandálico (sobre todo el grafiti barrista, el writing, el de consigna), o como algo artístico (los murales, el arte callejero); uno que daña y debe ser limpiado, otro que debe ser valorado, aunque también controlado. Esta posición refleja la vieja distinción entre el arte y el vandalismo en cuanto al grafiti. Es una forma de expresión cultural que atrae reacciones extremas, influidas por la teoría de la ventana rota donde la visibilidad de desorden se ve como legitimación para el comportamiento criminal (Wilson y Kelling 1982; Schacter 2008; Ferrell 1996). La participación de los grafiteros bogotanos ofreció otra perspectiva. German Gómez de la Secretaria de Cultura, Recreación y Deporte en Bogotá explicó que su punto de vista, y la dirección de la ley, cambiaron solo después de las conversaciones entre los grafiteros y los oficiales, sostenidas en las "mesas de grafiti". En estas mesas los grafiteros bogotanos reúnen con los representantes de la alcaldía, sobre todo el Instituto Distrital de las Artes, y discuten las oportunidades para proyectos de grafiti o los problemas enfrentados 
por artistas. Según Gómez, este diálogo ha tenido un gran impacto. Por ejemplo, en vez de intentar persuadir a los jóvenes con remplazar al bombing por la creación de murales y otras opciones más estéticas, dice que empezaron a entender que el bombing hace parte de la subcultura y del desarrollo creativo de los grafiteros.

Además, el contexto sociopolítico entorno a la elaboración de la ley de grafiti fue fundamental para que la alcaldía de Petro viera de manera favorable a los jóvenes y reconociera el grafiti como arte. Hasta cierto punto, el gobierno se encontró forzado a aclarar su posición frente al grafiti en Bogotá gracias al escándalo del caso de Diego Felipe Becerra y la atención mediática y pública que obtuvo. El 19 de agosto de 2011, Diego, un joven de 16 años, fue asesinado por la policía cuando hacía grafiti en el norte de Bogotá. La investigación por su muerte, que aún no ha concluido, está rodeada de numerosas alegaciones de corrupción e intimidación. El consenso actual sobre el caso reconoce que un policía le tiró dos veces por la espalda cuando intentó correr para escapar. La policía montó un encubrimiento, puso un arma en la escena del crimen y pagó a dos testigos falsos para que corroborasen la versión de los oficiales de que Diego y sus amigos habían robado un autobús. Mientras que la ley de grafiti estaba en curso, el caso contribuyó a cambiar el tono de la ley y la perspectiva de la alcaldía, así como la de la sociedad urbana. Los grafiteros no solo debían ser controlados, sino también protegidos. Que un joven pudiese ser asesinado solo por hacer grafiti y que la policía hubiese actuado de manera tan corrupta incrementó el nivel de debate público sobre el derecho de expresarse en la ciudad y los riesgos enfrentados por los grafiteros.
Las dinámicas del grafiti en Bogotá ofrecen un contexto en el cual se puede analizar la complejidad de la participación de los habitantes en la producción del espacio urbano. Por una parte, reflejan el deseo de Lefebvre, ya que los grafiteros reclamaban su derecho a la ciudad. Por otra parte, trabajar con los políticos no significa un cambio sistemático sino una negociación. La combinación de la necesidad y del deseo de responder al grafiti se puede entender utilizando la conceptualización de las relaciones entre el Estado y los habitantes de Partha Chatterjee. Propone Chatterjee que hay un espacio de negociación entre el Estado y los grupos marginalizados o excluidos de la sociedad civil. Estos últimos no son ciudadanos porque no tienen acceso a todos los beneficios de la ciudadanía y la participación en la sociedad y la política. Sin embargo, pueden reclamar derechos o compromisos dado su persistencia en ser presentes y visibles (Chatterjee 2004). A pesar de que el grafiti no va a ser legal en todos los espacios y que la legalidad tampoco es algo que concierne a los grafiteros, ellos sí tienen un poder colectivo y tienen sus exigencias, como, por ejemplo, la no violencia por parte de la policía. Con la ley de grafiti y las mesas distritales de grafiti, los grafiteros están participando en la política y la democracia de forma más tradicional que solo pintar la ciudad de manera transgresiva. La forma en que el gobierno reacciona a estas demandas refleja sus prioridades en cuanto a la sociedad urbana que quiere. El derecho a la ciudad es algo que celebra la incrementación del valor dado a los tradicionalmente excluidos, para hacer del espacio público algo más democrático, más centrado en los que utilizan la ciudad. En lo que sigue, investigo el concepto de apropiación para entender como los que hacen grafiti interpretan el derecho a la ciudad de forma más amplia que 
solo participar en la ley de grafiti, y cuáles son los obstáculos que les enfrentan.

\section{La apropiación de la ciudad y los límites del derecho a la ciudad}

La participación de los grafiteros en las dinámicas políticas del espacio público bogotano refleja una demanda para incluir la sociedad urbana, y sobre todo los grupos sociales más marginalizados, en las decisiones estéticas de la ciudad. Como he mostrado, para participar en la ciudad han tenido que negociar el derecho a la ciudad, lo que revela las complejas relaciones de poder entre los ciudadanos y el gobierno municipal. Sin embargo, lo que importa no es que el grafiti sea legal o ilegal, sino que representa la apropiación del espacio público por parte de los jóvenes. En lo siguiente, propongo que el grafiti apoya la posición filosófica de Lefebvre que estima el valor de uso y no el valor de cambio del espacio. Al reclamar el derecho a la ciudad en la vida cotidiana, los jóvenes evidencian una formación política a través de prácticas creativas basadas en la apropiación del espacio urbano. A diferencia de la participación, la apropiación se asocia más con la resistencia porque tiene una carga ideológica que reclama un cambio fundamental en la estructura social, en comparación con una mayor participación de ciudadanos en la misma estructura (Purcell 2002: 103). Las perspectivas de los grafiteros bogotanos entrevistados en este proyecto demuestran su agencia conforme con una geopolítica de la cotidianeidad. Sin embargo, la intención de apropiar el espacio público para crear una ciudad más democrática se enfrenta con la recepción de grafiti en Bogotá. Los límites del derecho a la ciudad se revelan cuando las reacciones a estas prácticas no estén acordes con la legitimación ya descrita. En la última parte del artículo, considero la reproducción de las desigualdades y exclusiones que refuerzan la jerarquía social en Bogotá a través de los imaginarios urbanos de grafiti.

\subsection{Apropiar el espacio urbano}

La visión de Lefebvre en cuanto al derecho a la ciudad tiene que ver con la utilización del espacio para la creatividad, para el encuentro. Como dicen Amin y Thrift, la ciudad democrática es

[U]n lugar de descubrimiento, y la realización de la posibilidad social, de la exploración democrática a través de los esfuerzos de los mismos ciudadanos, como agentes libres y socializados (Amin y Thrift 2002: 143). ${ }^{12}$

El espacio urbano debe ser valorado por las oportunidades que ofrece a los usuarios, a los habitantes de la ciudad. En cambio, la producción capitalista de espacios urbanos estima la urbanización en cuanto al valor de cambio. En general, el grafiti bogotano apoya la visión de la ciudad democrática, dado que un análisis del significado de hacer grafiti revela el valor de la apropiación cotidiana del espacio público. El grafiti tiene una carga simbólica para quienes lo hacen, y para entender el significado se necesita escuchar a sus creadores. Según ellos, es una manera de comprometerse con el espacio en su entorno, de habitar la ciudad de tal forma que uno ve y se integra en el espacio público y sus dinámicas sociales y políticas. La ciudad pintada por sus habitantes es, por lo tanto, una ciudad en donde la sociedad participa en su construcción, en vez de ser excluida y alienada. Lili Cuca lo describe así:

\footnotetext{
"a place of becoming, and the fulfilment of social potential, of democratic experimentation through the efforts of citizens themselves, as free and socialized agents"
} 
[E]l grafiti permitió que muchas zonas de Bogotá cambiaran y se transformaran a través de la imagen por, por como es el grafiti, o por como es el street art [...] que de verdad genera cambios dentro del espacio, en las dinámicas. Así sea solo mientras, por los tres días o la semana que la gente esté pintando, hay una alteración de la cotidianidad, del espacio. Pero todas estas cosas son como, estamos aportando también, a la cultura de un país. Porque somos los jóvenes quienes estamos haciendo esto. La gente joven quien se está apropiando de la ciudad de esta manera. ${ }^{13}$

La apropiación de la ciudad por parte de los jóvenes ha cambiado dos cosas para Lili Cuca, la estética de la ciudad y la vida cotidiana mientras están pintando. Las interacciones sociales entre los habitantes cambian alrededor del acto de pintar la ciudad, sobre todo cuando sea algo considerado bonito. Además, la perspectiva de los habitantes sobre la ciudad puede transformar al nivel del imaginario social, y el hecho de que son los jóvenes que lo hacen es significativo. Demuestra su acción política a través del espacio público y de escala cotidiana, pero aún más importante es el hecho de los jóvenes participen en, y construyen, la ciudad de manera autónoma y creativa. Así que, para Toxicómano, ser grafitero no se trata de su estilo ni del contenido de su trabajo, ni de la cantidad de murales o grandes obras que hace. Se trata de habitar la ciudad:

O sea, me molesta, lo que te decía, de la persona que pinta muy bien pero nunca ha pintado en la calle, de pronto tiene la oportunidad de pintar un muro y ya entonces, como que asume esa dinámica, pero todavía no ha, no se ha dado cuenta de todo lo que hay alrededor, que pintar en la calle no es subirse a un andamio, y ponerse audífonos y no ver qué sucede abajo. No, pintar en la calle es saber qué pasa en el barrio y estar integrado. Entonces, le pide uno a la gente que entra, siempre que es como parce, averigua un poco. $Y$ averiguar no es preguntarle cómo se hace cosa por cosa sino salir a probar y pintar. ${ }^{14}$

Entrevista con la autora

14 Entrevista con la autora
Para ser grafitero hay que apropiarse de la ciudad e integrarse a la vida cotidiana. Ocupar la ciudad es una manera de ver las dinámicas urbanas entre los habitantes, y entre ellos y el espacio en su entorno. Hay un aprendizaje dentro de la experiencia de pintar la ciudad. Para el grupo de arte callejero Dexpierte, pintar en la calle es algo más, es elegir estar integrado en la vida de la calle debido a las oportunidades políticas que ofrece.

\begin{abstract}
[E]s empezar a ocupar los espacios de tu propia ciudad, con un mensaje claro, político [...] porque es de nosotros, ¿sí? Como romper ese espacio público de que toda la gente está metida en los centros comerciales; también tiene otras cosas [...] Y poder estar en esa calle con tantas realidades que pasan en nuestro país, entonces es como también una experiencia personal, y obviamente con toda la intención política de dialogar con las personas. ${ }^{15}$
\end{abstract}

Su punto de vista expresa la conceptualización ofrecida por Andrzej Zieleniec, para quien el grafiti simboliza "un desafío al espacio normal, banal, operativo y cada vez más dominado por la mercantilización y la privatización", e insiste en que:

[L]o urbano se quede como lugar de encuentros, un foco y epicentro para la comunicación y la información, para las interacciones significativas y para la diferencia, para la diversidad y un sorprendente potencial creativo (Zieleniec 2016: 10). ${ }^{16}$

En otras palabras, insistiendo en que el espacio es social y que todo el mundo tiene derecho a participar en él: el espacio público es algo para disfrutar y utilizar, y no solo gastar dinero en él o evitarlo a causa del temor de los encuentros

15 Entrevista con la autora

16 "a challenge to normal, banal, functionalised and increasingly dominated commodified and privatised space", instead insisting that "the urban remains as a place of encounters, a focus and locus for communication and information, for meaningful interactions and for difference, diversity and for creative and surprising potential" 
desconocidos. Sobre todo, para Dexpierte la apropiación del espacio público significa una intervención en las dinámicas sociales porque representa la oportunidad para el diálogo político a través de prácticas creativas y artísticas. Como dice De Ruiter, hablando de grafiti en Atenas: "poniendo su arte en el espacio público, los artistas callejeros intentan transformar ambos la cultura y la política en algo común, en el sentido en que pertenece a todos" (De Ruiter 2015: 593). ${ }^{17}$

No hay espacio para detallar todas las perspectivas y opiniones políticas que tienen los grafiteros - algo imposible de todos modos porque cada uno es distinto - pero sí se puede mostrar que construyen sus políticas mientras que reclaman su derecho a la ciudad. Estas interpretaciones de lo valioso de hacer grafiti resaltan el papel del espacio urbano como lugar de encuentro y diálogo y la priorización del valor de uso. Ambos son formas de resistencia en una ciudad dominada por los intereses de las elites financieras y políticas. La apropiación del espacio es una manera de reclamar el derecho a la ciudad, de priorizar su valor de uso, de mostrar que la gente debe tener más control sobre lo que es el espacio y que puede pasar en ello (Purcell 2002: 103). El grafiti es una manera de tomar la iniciativa de apropiarse de los espacios públicos y urbanos para crear una ciudad que sea artística, política o de libre expresión, pero de todos modos compartida y gratis - una suerte de "urban commons" (Harvey 2013). Hablando de grafiti y arte urbano en el contexto internacional, Waclawek dice que "no es solo una manera de expresarse y de crear oportunidades para la comunicación

"by placing their art in public space, street artists seek to render both culture and politics common, in the sense that it belongs to everyone" en una ciudad, también, lo más importante, es que es una negociación de lo que significa la participación en la cultura visual de una ciudad" (Wacławek 2011: 159). ${ }^{18}$ Para Zieleniec, la participación de los grafiteros es "un ejemplo de retomar la ciudad como una 'oeuvre', un trabajo artístico vivo y creativo, perennemente en un proceso constante de creación y recreación" (Zieleniec 2016: 11). ${ }^{19}$ Por lo tanto, a pesar de que los grafiteros individuales tendrían perspectivas políticas distintas, sus acciones en cuanto a la apropiación del derecho a la ciudad es una subversión simbólica.

Si volvemos a la ley de grafiti y la legitimación de esta práctica urbana, parece que los jóvenes han tomado el derecho a la ciudad y han encontrado una manera de apropiarse del espacio público para valorar su uso creativo y democrático. En su forma de geopolítica de lo cotidiano, estos jóvenes resaltan las posibilidades de las acciones ciudadanas. La celebración del grafiti en la ciudad apoya esta legitimación y muchos artistas bogotanos hablan de buenas interacciones con los ciudadanos, sugiriendo que el espacio creativo representa un espacio para el diálogo democrático. Sin embargo, la realidad de hacer grafiti en Bogotá es compleja. Un enfoque en las dinámicas contemporáneas del grafiti en Bogotá desde la introducción de la ley demuestra también una continuación de la exclusión y la alienación.

\footnotetext{
18 "is not only a means of self-expression and a way to create opportunities for communication in a city, but also, most significantly, it is a negotiation of what participation in a city's visual culture represents"

19 "an example of a return to the city as oeuvre, a living creative work of art, always in the process of being made and remade"
} 


\subsection{La jerarquía estética}

A pesar del reconocimiento de grafiti en la ley, Gómez dice que hay un problema, una vieja tendencia de ver lo más artístico como legal y lo más visceral, lo más tradicionalmente político o directo, como ilegal:

\begin{abstract}
[L]a gente asume, en su sentido común, que lo artístico son los grandes murales que hay en la 26 , o los que se han hecho últimamente en el centro, ¿no? Entonces fijate que sí existe una hegemonía, en la opinión pública, en lo que se considera buen grafiti y el mal grafiti. Creo que eso fue un error de la administración, no haber trasmitido adecuadamente la información y plantear que el grafiti, digamos la práctica responsable de grafiti, no tiene que ver con la calidad estética del grafiti. Sino si tiene permiso o no tiene permiso. [...] [Y], en detrimento de eso, se ha afectado otro tipo de grafiti, como el tag, el writing, el político. [...] Luego ahí también hay una violencia simbólica porque los practicantes de otros tipos de grafiti se sienten excluidos por las mismas políticas administrativas o distritales. ${ }^{20}$
\end{abstract}

Aquí el imaginario urbano del grafiti en Bogotá es relevante. Como dice Gómez, el valor percibido de grafiti depende del estilo y no del reconocimiento que los jóvenes tengan el derecho de expresarse libremente. La imagen de los grafiteros ha sido influida por la ya mencionada teoría de la ventana rota y está relacionada con una imagen y posición política sobre qué es la ciudad y quiénes tienen derecho a ella. Como dice Rama, la condenación del grafiti ha sido históricamente mezclada con la necesidad de las elites de mantener el estatus social de los letrados:

[L]o que intentaba era conservar ese orden de los signos que es la tarea preciada de la ciudad letrada, la cual se distingue porque aspira a la unívoca fijeza semántica y acompaña la exclusiva letrada con la exclusiva de sus canales de circulación (Rama 1984: 55).
Así que la ciudad letrada excluye de manera activa a los grupos sociales vistos como indignos (o incapaces) de cultura, educación y, por lo tanto, de poder, en la medida en que no escriben de la manera legitimada para participar en tales instituciones. Los que sí escribieron en las paredes, los que hacían grafiti, sea con críticas sociopolíticas, obscenidades, o simplemente sus nombres, estaban etiquetados como "necios"; "'hombres de baja esfera', tanto por sus mensajes como por su torpe manejo de la escritura" (Rama 1984: 53-54). La continuación de esta jerarquía es visible en los discursos sobre el grafiti en Bogotá. Como dice DjLu:

Creo que eso sería una de las cosas, de los problemas digamos de la visibilización [sic] y de la legalización de la práctica. Que en ese tipo de institucionalización de una práctica salvaje [...] empieza a haber curadurías, empieza a haber segmentación, empieza a haber estratificación. Entonces eso es un poco lo que se está dando ahora. Que la gente empieza a tomar partido, a decir esto me gusta, esto no me gusta. Esto es válido, esto es feo, esto es ilegal, esto lo deberían prohibir, esto lo deberían apoyar. En últimas, eso es una cosa que está reforzada por las instituciones y por los medios, sobre todo. Muchas veces la editorial, el tinte editorial de los medios [...] va en el sentido de satanizar el grafiti y el tag, y adorar el arte urbano y el muralismo. $Y$ hay que entender que no es que sea uno bueno y otro malo. Son totalmente diferentes. Y a mi juicio, ambos son del carajo. Ambos son bacanísimos. ${ }^{21}$

Dentro de las subculturas del grafiti hay más respeto para las diferentes maneras de pintar la ciudad porque, en última instancia, pintar en la calle es reconocer que todos tienen el derecho a la ciudad. Así que las jerarquías vienen de afuera y, según DjLu, de las instituciones y los medios, sobre todo. Alexander Araya López dice que la prensa al describir esta expresión del arte urbano refleja ideologías políticas: 
Si los discursos en dichos medios (en este caso, la prensa escrita) presentan una práctica social de forma estereotipada o incompleta, o 'criminalizan' a sus productores, la posibilidad de generar cambios sustanciales hacia una democratización del espacio urbano se reduce. Sin embargo, cuando existe un debate sobre la legalidad o el derecho a la apropiación y uso del espacio, es posible esperar que los derechos de ciertas poblaciones sean visibilizados e incluso reconocidos (Araya López 2015: 96).

La imagen de lo que es el grafiti, y de quiénes lo hacen, es, por lo tanto, fundamental en cuanto al derecho a la ciudad. Por eso es necesario que la ley de grafiti reconozca la creatividad y la cultura de los practicantes, pero eso no implica necesariamente que haya cambiado la opinión pública.

El problema no es que haya opiniones diferentes sobre el grafiti, es que tal jerarquía estética refleja y reproduce una jerarquía social. Entrevistando a personas en universidades y en las calles de la ciudad, oí descripciones como "mamarracho", "desadaptado", interpretaciones de la práctica que la presentan como una "falta de respeto", "una falta de sentido de pertenencia" o "contaminación visual". Sobre todo, los tags y el grafiti de barrista fueron descritos como "cosas sin sentido", representaciones de "desorden" que no es "sano para la sociedad". En particular, con referencia a la imagen de grafiti de barrista mostrada en la introducción, un estudiante dijo: "para mí es un rayón. Eso lo hizo un ñero. Pues, a mi concepto, ¿no? O sea, un ñero, ¿a quién importa lo que ellos piensen?", mientras que otra persona dijo "eso no es una protesta, es gente sin oficio, gente que no tiene nada que hacer". En vez de dialogar con la gente, la jerarquía distingue entre el grafiti que tiene sentido y el grafiti sin sentido, desenmascarando las relaciones sociales y su reproducción en el espacio público. Toxicómano, por ejemplo, dice que hay muchas conjeturas falsas sobre qué significa hacer grafiti:

Entonces es como que, que es también que es un impulso ya de la gente en la calle, que le da un símbolo de 'mi ciudad y me siento cómodo acá, o sea eso me pertenece, esto es mío'. La gente dice 'uy estaba dañando' [...] u 'odian Bogotá' y creería que, al contrario, la gente que hace grafiti es amante de su ciudad, la conoce de arriba abajo, porque todo el tiempo lee sus rejas, sus puertas, sus ventanas, sus horarios. Está en otra dinámica totalmente diferente pero el problema es que algunos no les interesa establecer este diálogo. ${ }^{22}$

El diálogo es esencial para entender el significado y lo político de apropiarse de la ciudad a través del grafiti, pero falta cuando los sujetos sociales no están considerados como ciudadanos legítimos.

Pintando en la calle, uno siente el efecto de los imaginarios urbanos cotidianos de grupos sociales, del espacio público y de las formas de expresión cultural. Como dice Skore:

\begin{abstract}
Pues normalmente una gente le agrada cuando pinto pues legal, que son colores, quizás más estético, más una idea, pues, pensada. Pero es algo irónico que en ese momento ellos quizás se sienten con autoridad a decir, no me gusta, o cámbielo, o porque no hace algo bonito. Pero cuando uno está pintando bombing que te ven haciendo tags en la calle, te respetan demasiado, o te tienen miedo, nadie dice nada, entonces es como chistoso y controversial. Como que, uno está pintando algo bonito para la gente, que nadie le está pagando a uno, y sí critican. Y cuando uno está destruyendo la ciudad, entre comillas, te tienen miedo y respeto.
\end{abstract}

Estas experiencias representan otra perspectiva de la geopolítica de lo cotidiano. Hay una contradicción en las actitudes de las personas hacia el grafiti, que refleja la presencia del miedo en la vida cotidiana de Bogotá. Los que hacen grafiti experimentan este miedo a través de los estereotipos. Skore afirma:

\footnotetext{
Entrevista con la autora
} 
La gente los asimila con delincuencia, con ladrones, con gente armada. No conozco el primer que sea con estas características. Quizás por eso lo que te digo que es lo controversial que cuando salgo a hacer bombing la gente te respete y te tiene miedo. Pero es por un imaginario social. No es cierto, es como un miedo. Como te dije al comienzo, que la gente te dice que acá roban un montón, pero es un miedo social que cualquier persona te pide la hora, te va a robar. Así. Y me pasa, yo a veces soy ‘¿tiene hora?' y dice 'no, no', o ‘disculpe, ¿dónde queda tal cosa?' y 'no, no sé'. Y se encierren en su mundo.'23

Estas perspectivas develan tanto lo político de hacer grafiti en el espacio público, como también las posiciones ideológicas que intentan negar su agenda política. Cuando las actividades políticas no están reconocidas por la hegemonía, hay una deslegitimación de prácticas juveniles porque no participan en la política de manera tradicional, pero sí lo hacen de otras formas, incluidas la desobediencia y prácticas corporales en el espacio, y sí tienen ideas sobre la política (Patiño et al. 2014). Las descripciones estereotipadas criminalizan o deslegitiman el significado del grafiti, de todos modos, ciegas a las motivaciones de quienes los hacen. La descripción de sociedades urbanas por Manuel Delgado es útil aquí, aunque esté hablando de Barcelona, porque interpreta la representación de ciudadanos como buenos o malos a través de una jerarquía social:

Parece que esas bolsas crecientes de ingobernabilidad se nutren de lo que se mencionaba como las nuevas 'clases peligrosas', aquellas que el nuevo higienismo social, como el del siglo xix, clama por ver neutralizadas, expulsadas o sometidas a toda costa: los jóvenes, los drogadictos, las prostitutas, los mendigos, los inmigrantes sin alojamiento. $Y$ es así que muchas de las actuaciones en materia de promoción de la 'civilidad' y la 'ciudadanía' responden hoy al hecho de que a políticos y promotores les parezca inaceptable que, en plena ciudad, a la vista de todos, aparezcan las evidencias de las nuevas y las viejas formas de miseria, elementos escasamente decorativos que pueden asustar a los turistas y los propietarios e inquilinos, a los que se pretende atraer a toda costa (Delgado 2012: 63-64).

Su punto es que no se trata de la bondad o maldad de los ciudadanos, sino de los intentos de ciudadanos y administraciones de no pensar en la violencia capitalista y sistemática que excluye y reproduce la alienación, y el no pensar tiene la forma de denigrar a los que utilizan el espacio urbano de "otras" maneras. La proximidad a la calle es algo que, para Stinkfish, explica por qué hacer grafiti es particular, es distinto a ser artista. Dice que están más cerca al mundo de la calle, de la cotidianeidad del espacio urbano normalmente mal visto:

\begin{abstract}
Hay un montón de realidades, el grafiti muestra otra que está en paralelo. Y es más cercana a todo este montón de cosas que pasan en la calle, como así los vendedores ambulantes, como toda la vida que está en la calle, que para muchas personas es algo súper lejano, prohibido, sucio, feo, ilegal, es algo más cercano a nosotros, ¿sí? Entonces por esto te digo que el grafiti es autosuficiente, lejos del arte, del diseño, ¿sí? La vida de un artista por lo general, o de un diseñador, está más cercana a este mundo legal. Nosotros estamos más cercanos a la gente que venden cosas en la calle, a la gente que pega carteles, a todo lo que puede suceder en la calle, que supuestamente no cumple con ciertas normas. ${ }^{24}$
\end{abstract}

Stinkfish asocia las reacciones al grafiti al sistema capitalista que interpreta la legitimidad de la creatividad a través del valor de cambio:

Tienes una lógica de vida donde lo gratis es sucio, donde si alguien hace algo por su cuenta, no vale. ¿Sí me entiendes? Si a mí me llevan a un barrio de dinero, y me pagan una grúa, y me toman fotos, así se salen todos los del barrio, muchos del barrio, como ahh tú quién eres, estás grabando algo para la televisión, ¿sí? Mientras si yo lo hago por mi cuenta, que pasa todo el tiempo, que voy, pinto paredes y llevo mis cosas no lo digo a nadie, no tiene que ver con nadie, todo el mundo para y preguntan, pero ¿quién le pagan, o si lo paga el gobierno, o el dueño

\footnotetext{
Entrevista con la autora
} 
de la casa? O, ¿usted por qué hace eso, por dónde usted sacó la plata? [...] Entonces yo creo que tiene que ver más con esa lógica de que si no estás recibiendo dinero, es algo raro [...] sospechoso, mal hecho. ${ }^{25}$

La ideología influye la selección de quiénes deben tener visibilidad en la ciudad, quiénes pueden participar en el espacio público y cómo. Si el derecho a la ciudad concebida por Lefebvre celebra la apropiación del espacio público y la promesa de la libre expresión, la perspectiva de Stinkfish niega la visión de Bogotá como ciudad democrática. En vez de apoyar la libre expresión de todos los ciudadanos, hay una selección de los buenos ciudadanos a través de la jerarquía estética. Como dice Ferrell, en los intentos de controlar el espacio urbano, lo visual es crucial: "en la ciudad ocupada, los de afuera y sus culturas están expurgadas, 'desaparecidos', haciéndolos invisibles e inaudibles" (Ferrell 2001: 227). ${ }^{26}$ Visto de esta manera, el espacio urbano no solo expresa los múltiples públicos y sus formas de participar en la ciudad y expresarse, es también un espacio de contención ideológica y de la reproducción de exclusión.

El grafiti es un ejemplo visible de la heterogeneidad de la sociedad urbana, de los diversos públicos y sus diversas maneras de habitar o utilizar la ciudad. Además, es un ejemplo de la geopolítica cotidiana como forma de resistencia, dado que reclaman el derecho de transformar la ciudad. Sin embargo, las reacciones hacia el grafiti también reflejan las relaciones de poder en la vida cotidiana y el control sobre el espacio público y la sociedad urbana. La jerarquía estética revela los límites

\footnotetext{
Entrevista con la autora

26 "in the occupied city, outsiders and their cultures are expurgated, 'disappeared,' rendered invisible and inaudible"
}

del derecho a la ciudad, basados no en la necesidad de mantener el espacio público limpio sino de controlar las expresiones de ciudadanos señalados como (in)aceptables. El hecho de que celebran el grafiti que sea estéticamente agradable pero no el que critica o denuncia sugiere que hay un intento de no reconocer las realidades de alienación y marginalización.

\section{Conclusión}

Con el anterior análisis de grafiti en Bogotá he intentado mostrar las complejas negociaciones e interacciones entre el Estado y los ciudadanos en lo que concierne el derecho a la ciudad. En cuanto al Estado, la ideología y la priorización de distintos grupos sociales afectan las políticas sobre el espacio: cómo se utiliza y quienes pueden utilizarlo. Sin embargo, la ciudadanía no es pasiva - busca activamente su reconocimiento y sus derechos. Hasta qué punto el gobierno escuche a esta ciudadanía depende del momento específico - tal cual se puede ver con la ley de grafiti en Bogotá y la confluencia de las exigencias de los grafiteros con la disposición política de la alcaldía de Gustavo Petro.

La relación formal entre el gobierno y los grafiteros ofrece un ejemplo de la negociación al derecho a la ciudad. Al mismo tiempo, la participación en, y la apropiación de, los espacios de la ciudad no necesariamente conforme con la visión hegemónica del buen uso del espacio público. Hacer grafiti es una manera de expresarse políticamente, y las perspectivas y opiniones de los grafiteros entrevistados aquí revelan la participación activa de los ciudadanos en no solo transformar la ciudad estéticamente sino también en conceptualizar teorías del espacio 
público. Una teoría básica es que la ciudad debe ser apropiada por los que la habitan, aun si hay una confrontación con la ley.

Para complejizar el derecho a la ciudad aún más, la lucha no se encuentra simplemente entre la ciudadanía y el Estado. El derecho a la ciudad exigido por los grafiteros afronta las perspectivas de otros ciudadanos, quienes denuncian el grafiti. Estas denuncias no se limitan al desdén hacia pintar la ciudad sino a las identidades sociales que amenazan el "buen orden" de la vida urbana. La jerarquía social puede ser mapeada sobre una jerarquía estética, porque los ciudadanos entrevistados aquí no desdeñan a todos los tipos de grafiti, sino a los que representan las expresiones de los "sin respeto", que "no tienen nada que hacer", como el grafiti writing o el bombing.

Mi argumento no es que todo el mundo debe gustar de todo tipo de grafiti, sino que la producción y recepción de grafiti demuestra las complejas y discordantes actitudes frente a lo que significa vivir en la ciudad y expresarse mediante los espacios de la ciudad. Un enfoque en la geopolítica de lo cotidiano pone atención a las voces de los habitantes y a la diversidad de sus prácticas y perspectivas, reflejando el hecho de que son sujetos políticos y críticos, aunque no se expresan de la misma manera que los sectores académicos o las autoridades del gobierno.

\section{Bibliografía}

Amin, A, y Thrift, N J. 2002. Cities : reimagining the urban. Cambridge: Polity.

Araya López, A. 2015. "Discursos sobre las prácticas del graffiti en el periódico La Nación (2001-2010)". Ciencias Sociales 150: $91-138$

Attoh, K. 2011. "What kind of right is the right to the city?". Progress in Human Geography 35: 669-85.

Brenner, N., Marcuse, P. y Mayer, M. 2012. Cities for people, not for profit : critical urban theory and the right to the city. London: Routledge.

Busquet, G. 2013. "L'espace politique chez Henri Lefebvre: I'idéologie et l'utopie". Justice Spatiale : Spatial Justice 5: 1-12.

Chatterjee, P. 2004. The politics of the governed: reflections on popular politics in most of the world. New York: Columbia University Press.

Crehan, K. 2016. Gramsci's common sense : inequality and its narratives. Durham: Duke University Press.

De Certeau, M. 1980. "On the Oppositional Practices of Everyday Life". Social Text 3: 3-43.

De Ruiter, A. 2015. “Imaging Egypt's political transition in (post-)revolutionary street art: on the interrelations between social media and graffiti as media of communication". Media, Culture \& Society 37: 581-601.

Delgado, M. 2012. "El mito del espacio público: retórica ciudadanista y control social en Barcelona". Fricciones sociales en ciudades contemporáneas. Salcedo, M. T. y Salcedo Fidalgo,
A. (Eds.), Bogotá: ICANH. 61-82.

Dowler, L. y Sharp, J. 2001. "A Feminist Geopolitics?". Space and Polity 5: 165-76.

Ferrell, J. 1996. Crimes of style : urban graffiti and the politics of criminality. Boston: Northeastern University Press. 2001. Tearing down the streets: adventures in urban anarchy. Basingstoke: Palgrave.

Harvey, D. 2013. Ciudades rebeldes: Del derecho de la ciudad a la revolución urbana. Madrid: Ediciones Akal.

Hörschelmann, K. y El Rafaie, E. 2014. "Transnational citizenship, dissent and the political geographies of youth". Transactions of the Institute of British Geographers 39: 444-56.

Hyndman, J. 2004. "Mind the gap: bridging feminist and political geography through geopolitics". Political Geography 30722.

Kallio, K. P., Mills, S. y Skelton, T. 2016. Politics, Citizenship and Rights. Singapore: Springer.

Koopman, S. 2011. "Alter-geopolitics: Other securities are happening". Geoforum 42: 274-84.

Lefebvre, H. 2008. Critique of everyday life. London: Verso. 2014. The urban revolution. Minneapolis: University of Minnesota Press.

Lefebvre, H., Kofman, E. y Lebas, E. 1996. Writings on cities. Oxford: Blackwell.

Marcuse, P. 2012a. "¿Qué derecho para qué ciudad en Lefebvre?". Tribuna/Tribune 2: 17-21. 
2012b. "Whose right(s) to what city?". Cities for People, Not for Profit: Critical Urban Theory and the Right to the City. Brenner, N., Marcuse, P. y Mayer, M. (Eds.). London: Routledge. 24-41.

Muñoz, G. 2000. "Otra década perdida: Políticas Públicas de Juventud en la Región Andina”. Revista Nomadas 13: 239-46.

Pain, R. y Smith, S. J. 2008. Fear : critical geopolitics and everyday life. Burlington: Ashgate.

Patiño, J., Alvarado, S. V. y Ospina-Alvarado, M. C. 2014. "Ampliación de sentidos sobre las prácticas políticas de jóvenes con vinculación a siete movimientos sociales en Colombia". Revista Latinoamericana de Ciencias Sociales, Niñez y Juventud 12: $257-75$.

Purcell, M. 2002. "Excavating Lefebvre: The right to the city and its urban politics of the inhabitant". GeoJournal 58: 99-108.

Rama, A. 1984. La ciudad letrada. Hanover, N.H.: Ediciones del Norte.

Rincón, O. y Hoyos, M. P. 2013. "A Bogotá le encanta la independencia". Nueva Sociedad 243: 131-41.

Sarmiento Anzola, L. 2006. El infortunio de las políticas públicas de juventud en Bogotá. Bogotá: Ediciones Desde Abajo.

Sassen, S. 2013. "Does the City Have Speech?". Public Culture 25: 209-21.

Schacter, R. 2008. "An ethnography of iconoclash: An investigation into the production, consumption and destruction of street-art in London". Journal of Material Culture 13: 35-61.

2014. "The ugly truth: Street Art, Graffiti and the Creative City". Art \& the Public Sphere 3: 161-76.

Silva, A. 1987. "La perspectiva estética como estrategia comunicativa en ciudades Colombianas". Estudios sobre las Culturas Contemporáneas 1: 123-44. Diálogos 23. 1989. "La ciudad como comunicación". Revista 2006. Imaginarios Urbanos. Bogotá: Arango Editores.

Skelton, T. 2010. "Taking young people as political actors seriously: opening the borders of political geography". Area 42: 145-51.

Thibert, J. y Osorio, G. A. 2014. "Urban Segregation and Metropolitics in Latin America: The Case of Bogotá, Colombia". International Journal of Urban and Regional Research 38: 131943.

Tickner, A. 2008. "Aquí en el Ghetto: Hip-hop in Colombia, Cuba, and Mexico". Latin American Politics and Society 50: 12146.

Wacławek, A. 2011. Graffiti and Street Art. London: Thames \& Hudson.

Wilson, J. y Kelling, G. 1982. "The Police and Neighbourhood Safety: Broken Windows". Atlantic March 1982: 29-38.

Zieleniec, A. 2016. "The right to write the city: Lefebvre and graffiti”. Environnement Urbain/Urban Environment 10: 1-20. 
